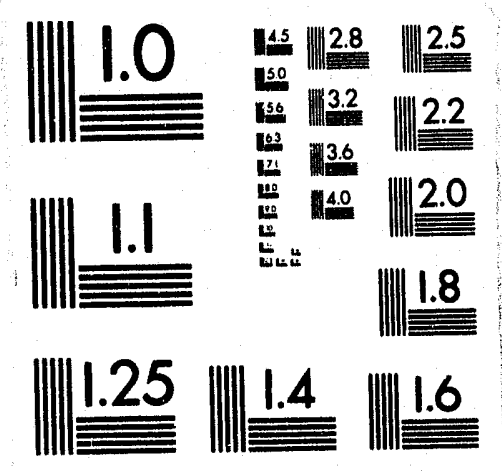



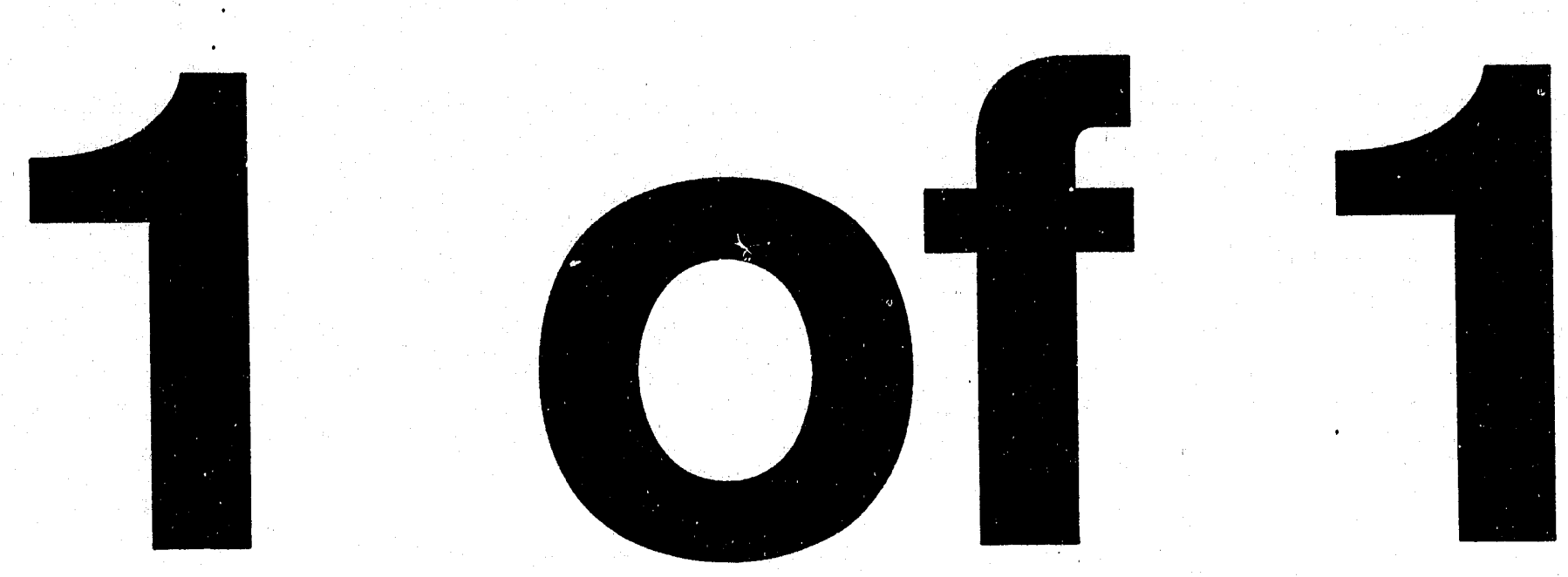


\title{
A Proposal For Reverse Engineering CASE Tools to Support New Software Development
}

\author{
Amelia Maxted \\ Advance Information Systems Department \\ Sandia National Laboratories \\ Albuquerque, NM 87185-5800
}

\begin{abstract}
Current CASE technology provides sophisticated diagraming tools to generate a software design. The design, stored internal to the CASE tool, is bridged to the code via code generators. There are several limitations to this technique: 1) the portability of the design is limited to the portability of the CASE tools, and 2) the code generators offer a clumsy link between design and code. The CASE tool though valuable during design, becomes a hinderance during implementation. Frustration frequently causes the CASE tool to be abandoned during implementation, permanently severing the link between design and code. Current CASE stores the design in a CASE intemal structure, from which code is generated. The technique presented herein suggests that CASE tools store the system knowledge directly in code. The CASE support then switches from an emphasis on code generators to employing state-of-the-art reverse engineering techniques for document generation. Graphical and textual descriptions of each software component (e.g., Ada Package) may be generated via reverse engineering techniques from the code. These reverse engineered descriptions can be merged with system overview diagrams to form a top-level design document. The resulting document can readily reflect changes to the software components by automatically generating new component descriptions for the changed components. The proposed auto documentation technique facilitates the document upgrade task at later stages of development, (e.g., design, implementation and delivery) by using the component code as the source of the component descriptions. The CASE technique presented herein is a unique application of reverse engineering techniques to new software systems. This technique contrasts with more traditional CASE auto code generation techniques.
\end{abstract}




\subsection{Introduction}

This paper presents a Computer Aided Software Engineering (CASE) environment that has been successfully employed in the development of a satellite ground system. This satellite ground system was developed in the Ada programming language. Traditional object-oriented design techniques were used in the design of the software.

Employing the documentation technique presented herein a design document that presents an accurate abstract toplevel view of the software was constructed. The CASE technique presented herein was accomplished via nonstandard use of commercial CASE tools.

\subsection{Motivation}

A software system is composed of design, code and documentation. CASE tools are directed at providing a single system description from which code and documentation can be generated. This system description contains both design and implementation details. CASE tools that accomplish this consistent view of the software system via code generators are prevalent. Current CASE technology provides sophisticated diagraming tools to generate a software design. These same tools provide facilities to specify implementation details. The system description (design and implementation) is bridged to the code via code generators. The premise is that the entire system, design and implementation, is defined in terms of the CASE "language" and stored via a storage structure defined internal to the CASE tool. From this intemal CASE storage, both code and documentation may be generated.

There are several limitations to this technique:

$\checkmark \quad$ The portability of the system is limited to the portability of the CASE tool.

$\checkmark$ Implementation is slowed down since all changes must be made via the CASE tool.

$\checkmark$ Maintenance is more complicated since it must be performed within the CASE tool environment.

$\checkmark$ Software acquired independently of the CASE tool can not be automatically integrated into the CASE system description.

$v \quad$ Bridging between the system description and the code, via Code Generators, is a significant and constant overhead.

$\checkmark$ Code generated by code generators is not trusted by software developers.
The CASE tool, though valuable during design, becomes a hinderance during implementation. Frustration frequently causes the CASE tool to be abandoned during implementation, permanently severing the link between design and code.

\subsection{Organization}

An overview of the internal implementation of both the traditional CASE based on code generators and the proposed CASE based on document generators is presented in Section 2.0, CASE Technology.

Details on the proposed CASE technique is provided in Section 3.0, The Proposed CASE Technique.

The CASE technologies are compared in Section 4.0, Comparison of the CASE Technologies.

New CASE capabilities that can solve some of the problems associates with current CASE capability are identified in Section 5.0, CASE Tools.

\subsection{CASE Technology}

The goals of CASE include:

v Providing graphical views of the system. These graphical views aid both the designer and the customer in understanding the system.

$\checkmark \quad$ Providing a bridge between the design and code.

Both of the comparing CASE techniques employ graphical editors for capturing design information as well as reverse engineering tools to graphically illustrate code. The main difference between the two techniques is that:

$\checkmark$ CASE based on code generators stores the system description via an internal storage format.

$\checkmark$ CASE based on document generators stores as much of the system description as possible directly as code.

Each of these CASE technologies are briefly described below. 


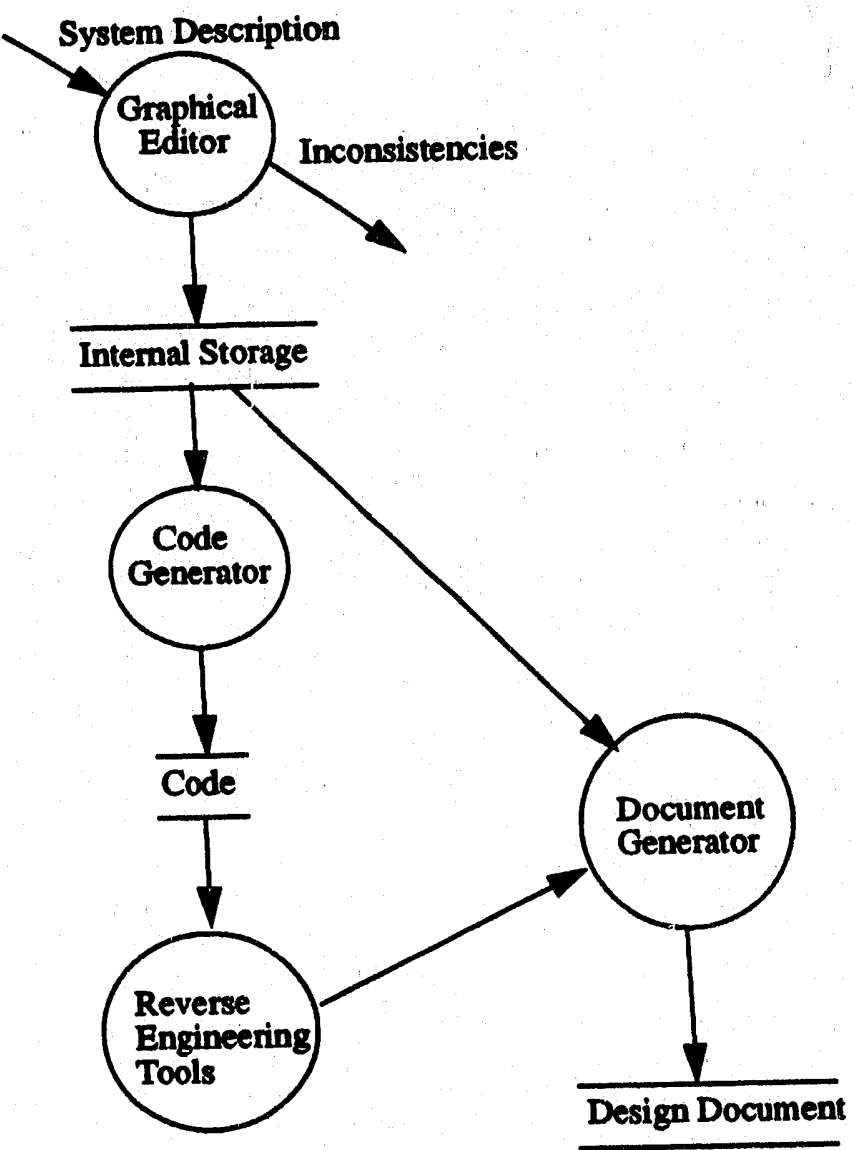

FIGURE 1: Today's CASE is Basod on Codo Gonorators

\subsection{CASE Based on Code Generators}

The CASE tools of today provide both code generators and reverse engineering capability. These tools are based on an internal storage structure for storing software system information. The data flow diagram on FIGURE 2: on page 3 illustrates the lasic internal structure of Today's CASE tools. The designer enters the system description via a graphical editor. This information is stored via a CASE internal storage structure. One of the more valuable functions provided by the CASE tool is consistency checking. The CASE tool reports inconsistencies in the system description.

To construct the code the designer executes the code generator. Many of Today's CASE tools also offer a set of reverse engineering tools. These tools construct graphical images of the code such as with-hierarchies and declara-

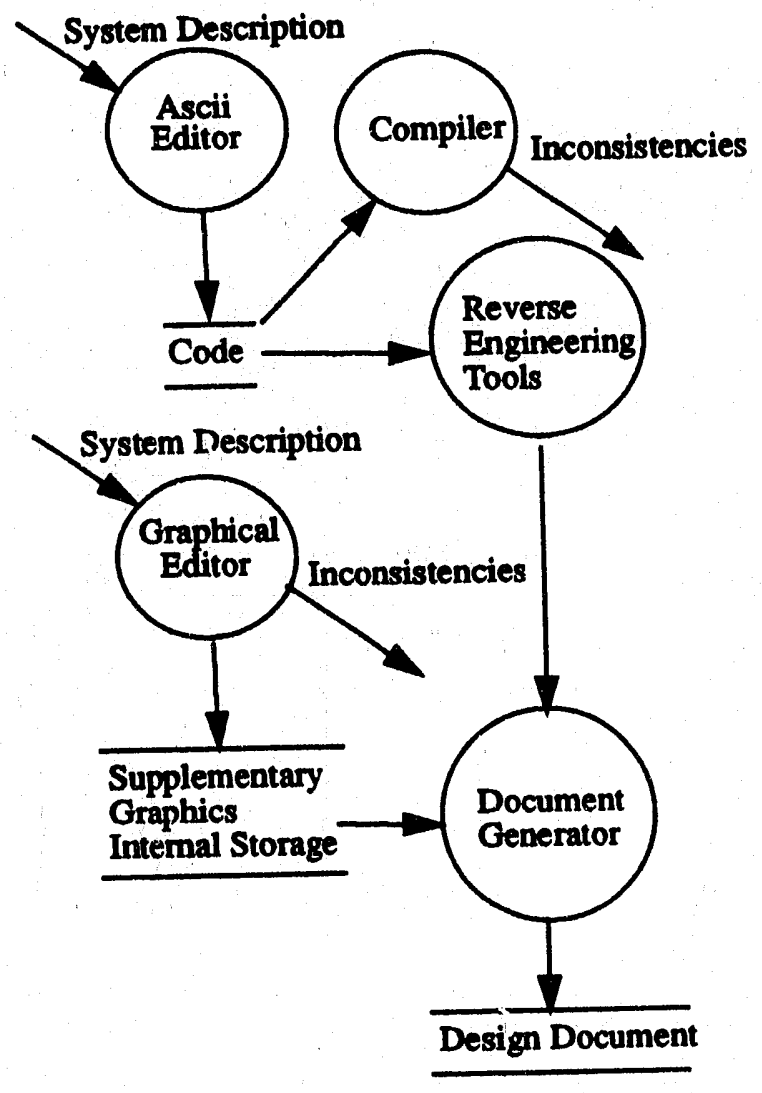

FIGURE 2: Proposed CASE ls Based on Document Conorators

tion diagrams [1]. The CASE tools provide facilities to generate graphics of the software solution. Most CASE tools depend on a document tool stich as FrameMaker to merge the graphics into a cohesive design document.

\subsection{CASE based on Document Generators}

An alternate approach is proposed herein. CASE based on document generators is illustrated in FIGURE 2: on page 3. The actual code product forms part of the internal CASE storage. This technique divides the internal CASE storage into two parts:

1. Textual information that can be stored directly in code, and

2. Graphical information that either is not available or can not easily be extracted from the code. 
An ASCII editor is used to generate the code. System descriptions stored in code include both textual descriptions stored in comments as well as the code itself. These textual descriptions include data dictionary entries and requirements allocated to the individual software components.

Graphical editors, such as those provided with Today's CASE tools, are employed to generate the supporting graphics. The supporting graphics are maintained separate from the code within the CASE Internal Storage. Supporting graphics include:

$\checkmark$ Overview diagrams, illustrating the interaction between components, and

v Graphical descriptions that can not be constructed via reverse engineering techniques such as state transition diagrams

Depending on the chosen methodology these overview diagrams might include overview Information Models [2] or Data Flow Diagrams (DFDs) [3].

Since the design is maintained directly in code, code generators are not employed. Document generators, based on reverse engineering techniques are employed to extract graphics and textual descriptions from the code. The document generators merge the reverse engineered descriptions with the supporting graphics to form a design document. Reverse engineering tools, provided by Today's CASE tools, may be used to create graphical descriptions of the code. These reverse engineering tools may also extract select text descriptions and/or requirements from the code comments. Merging the supplementary graphics with the graphics and text reverse engineered from the code is not fully supported by Today's CASE tools, but can be accomplished via document generators such as FrameMaker.

The CASE tool performs consistency checking on the overview diagrams, and the compiler performs consistency checking on the code. It would be relatively straight forward to create a tool that performs consistency checking between the code and the overview diagrams. Such a tool is not currently available. This consistency checking could also be extended to other supporting graphics such as state transition diagrams.

\subsection{The Proposed CASE Technique}

The proposed CASE technique requires the designer to separate the system description into two parts: code and supporting graphics. The supporting graphics consist of:

$\checkmark$ Overview diagrams, which illustrate the interaction between software components, and

v Graphical descriptions of the components, which are not readily available via reverse engineering techniques such as state transition diagrams.

The developer follows the following steps when emplo ing the proposed CASE technique:

$\checkmark$ Overview diagrams are constructed to identify the software components and illustrate the interaction between the components.

v Software component skiletal code is created for each identified component. The skeletal code is expanded to reflect the design knowledge on a component.

$\checkmark$ Descriptions of the components are constructed. Where ever possible these descriptions are constructed via reverse engineering techniques.

$\checkmark$ The supporting graphics are merged with the reverse engineered descriptions to create a design document.

$\checkmark$ As design transitions into implementations new implementation details are added directly to the code. The designer updates the supplementary graphics to reflect design changes.

Each of these steps are described in the following sections.

\subsection{Overview Dlagrams}

All designs begin with a set of diagrams that provide an overview of the system. For a detailed design, the overview diagrams identify major software components and illustrate the interaction between these components. The diagrams reflect the engineering design of dividing the software into software components.

The proposed auto document generation techniques may be applied to any methodology. A CASE tool, which sup- 


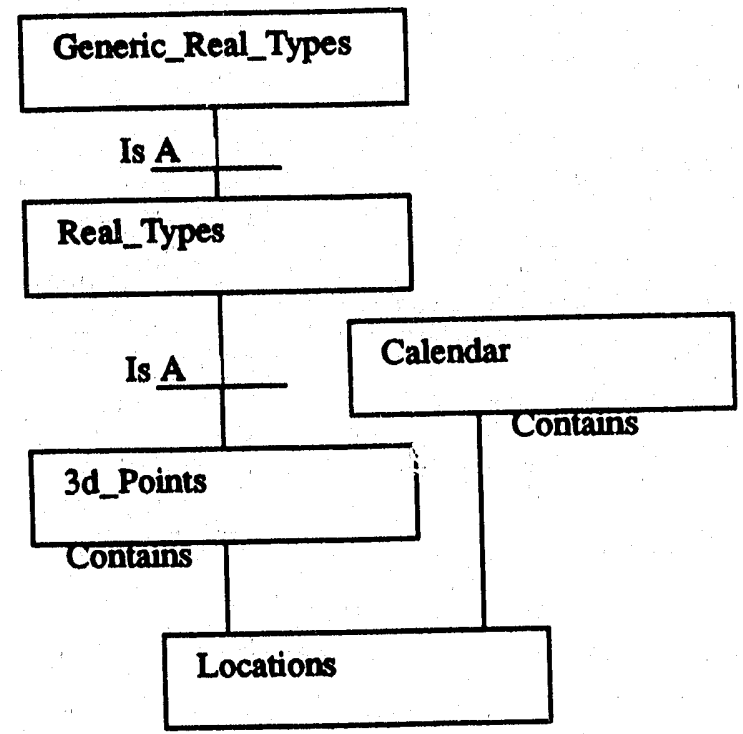

FIQURE 3: Overviow Information Model as an Example Overviow Diagram

ports the selected methodology, is employed to generate the overview diagrams. Typical overview diagrams are Information Models and Data Flow Diagrams (DFDs),. Reverse engineering techniques are not used to generate the overview diagrams. The assumption has been made that the items identified on the overview diagrams map to actual software components.

Consider the example of defining a location in time and 3 dimensional space. This software component, Locations, is constructed from other components as illustrated on FIGURE 3: on page 5. This overview diagram in an Overvie w Information Model that identifies 5 classes and the interaction between them. Each class identifies a software component. The identified software components are:

v Generic_Real_Types - This software component exports the type Real_Vector as well as the Type Real_Matrix. Fundamental operations on both these types are provided.

$\checkmark$ Real_Types - An instantiation of Generic_Real_Types.

v 3d_Points - Defines a subtype of the type Real_Vector that has only three components.

$\checkmark$ Calendar-Exports a date-time stamp.

$\checkmark$ Locations - Defines a point in time and 3 dimensional space.
FIGURE 3: on page 5 is an overview information model. Details on the components such as attributes and methods have been suppressed. These details are deferred to the software component skeletal code. Details on the components appearing on the overview diagrams are defined twice, once in code and once on the overview diagrams. A CASE tool that detects and reports discrepancies between the code and the diagrams would minimize the problems with duplicating this information. Such a tool is not available to date. It is recommended that the detail on the overview diagrams be minimized, thus reducing the size of the duplication problem. For DFDs, this might mean suppressing annotations that indicate the persistence or concurrency of a component. For information models, this means suppressing the attributes and methods associated with a software component. This separation of overview from details results in overview diagrams that are simpler, less detailed, than traditional overview diagrams. As software components are identified, the job of generating skeletal code begins.

\subsection{The Software Component Code}

During the design phase, software components are identified. Once the coinponent is identified skeletal coding begins. To capture the design information directly in code, the designer generates skeletal code for each identified component. This skeletal code consists of a declaration and end only. The skeletal code serves as a place holder for design information. As information on the component becomes available, the skeletal code expands. Skeletal code during design evolves as described below:

1. The skeletal code is created containing no more than a declaration and end.

2. A textual definition of the component is provided as code comments. The comments should be encoded to indicate that they define a data dictionary description.

3. As the designer studies the objects, attributes are associated with the objects. These attributes are captured directly in code as type declarations.

4. Early in the design phase the designer may wish to map requirements to the software components. Requirements mapped to software components may be derived requirements satisfying a part of an overall system requirement. Textual mapped requirements may be placed as comments in the skeletal code. The com- 
ments should be encoded to indicate that they define the requirements.

5. As the design progresses, methods (subprograms) are associated with each object. The methods are captured directly in code as subprogram declarations. Initially, these subprogram declarations may be skeletal, void of parameters. Parameters are added only as knowledge on the methods (subprograms) increases.

Placing textual descriptions such as data dictionary entries and requirements in the code comments tends to complete the code product.

An example Abstract Data Type, defining the Class, Location, is illustrated on FIGURE 4: on page 6. This class of object specifies the location in time and 3 dimensional space. Component textual descriptions are provided as comments embedded in the code. The numbers on the left margin map each code statement to one of the design steps listed above. Arr example of a graphical illustration of the component that might be reverse engineered out of the code is illustrated on FIGURE 5: on page 6. The example graphical description is an information model. Other graphical descriptions that can be constructed from code

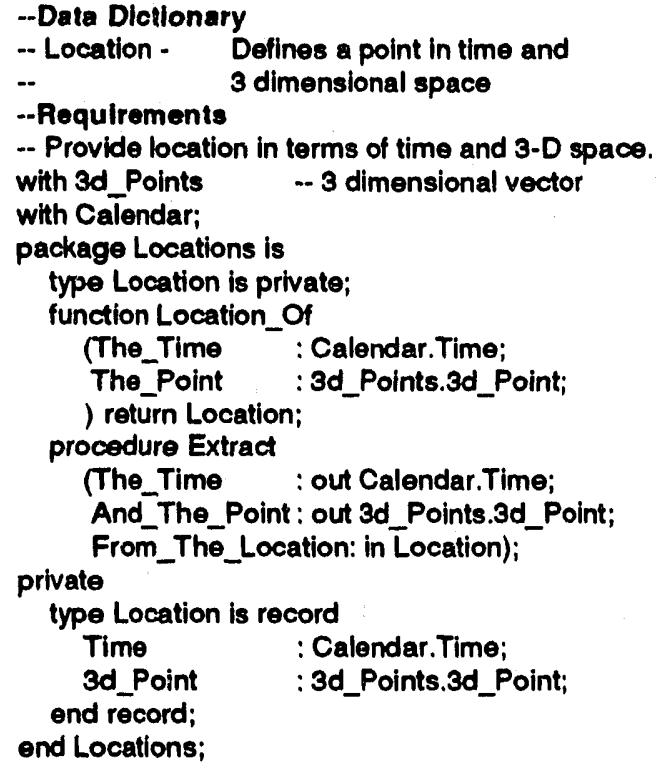

FIGURE 4: Example Ada Code Specification include invocation hierarchies, with-hierarchies and declaration diagrams.

\subsection{Other Supporting Graphics}

Where ever possible graphical representations of the software components are reverse engineered out of code.

Overview graphics are constructed to illustrate the interaction between these components. But there may be a need for additional supporting graphics. The other supporting graphics consist of:

$\checkmark$ Supplementary graphical descriptions, which describe the component but do not reflect the code. Since these descriptions are graphical they can not be embedded in the code comments and since they do not reflect the code they can not be constructed via reverse engineering techniques.

v Supplementary graphical is escriptions, which reflect the code, but reverse en gineering techniques to construct the graphical representation are not readily available, e.g., state transition diagrams.

\begin{tabular}{|l|}
\hline $\begin{array}{l}\text { Locations } \\
\text { Location - Defines a point in time and } 3 \\
\text { dimensional space }\end{array}$ \\
- Attributes - \\
Location (Time, 3d_Point) \\
\hline - Methods - \\
Extract
\end{tabular}

FIGURE 5: Example Graphical Description of Software Component Reverse Engineered out of Code 
Placing component descriptions in code comments restrict these descriptions to ascii text. If this restriction is not satisfactory then the comments can include a reference to supporting graphics. Supporting graphics may be needed to complete the descriptions.

Most of the graphics illustrating a software component can be constructed directly from the code via reverse engineering techniques. If the diagram can not be generated then supporting graphics that illustrate the software component are needed. These graphics tend to duplicate the code. This duplication problem is not resolvable by currently available CASE tools. Ultimately reverse engineering capability need to be expanded to support all graphics.

Specifically, CASE capability to reverse engineer state transition diagrams is needed.

Existing CASE tools may be employed to generate supporting graphics, such as state transition diagrams.

\subsection{Design Document}

The design document is constructed from:

v The supporting graphics, and

v The reverse engineered descriptions, text and graphics, of the software components.

FIGURE 3: on page 5 and FIGURE 5: on page 6 would both contribute to the design document.

The design document can readily reflect changes to the code by regenerating the reverse engineered descriptions.

Today's CASE tools do not provide the capability of merging the supporting graphics and reverse engineered descriptions into a cohesive design document. This task can be accomplished via a document generator such as FrameMaker[4].

\section{5 implementation}

The developer adds implementation details directly to the code. The skeletal codes are transformed into completed code products.

The developer must reflect design changes in the supporting graphics. These are major changes such as the addition or teletion of a software component or a change in the interaction between software components. This task may be minimized by:

1. Keeping the overview diagrams free of details, and

2. Employing a proposed tools that would report inconsistencies between the code and the supporting graphics.

The supplementary graphics are a small portion of the overall system, since the details are deferred to the code. Even without a tool to flag discrepancies, keeping the overview document up to date is a manageable task.

\subsection{Comparison of the CASE Technologies}

The major difference between these techniques is how the system description is stored. For CASE based on code generators, the CASE internal storage is the "bible" where the system description is stored. The code is generated from this internal storage. All changes must be made via the graphical editors supplied by the CASE tool. The graphics, created via the CASE tool form the design document.

For CASE based on document generators, most of the system description is stored directly in code. Supporting graphics supplements the code. The developer generates the supporting graphics via a graphical editor. Changes to the code are made directly via an ASCII editor. Both the supplementary graphics and the code define the system. Since much if not sil of the infiormation in the supporting graphics duplicates the code, techniques are needed to maintain consistency between these two views.

Both techniques offer graphical editors to define the software system. Both techniques employ reverse engineering tools to document code. But there are major differences in design, implementation and maintenance phases of development. Today's CASE tools, based on code generators, excel during the design phase. The benefits of the CASE based on document generator technique, are reaped as soon as the software development transitions into implementation.

The two CASE technologies are compared in the following sections. A summary of the results of this comparison is illustrated on FIGURE 6: on page 7. 
Code Document

Generator Generator

\begin{tabular}{l|c|c|}
\hline Design & + & - \\
\hline Implementation & - & + \\
\hline $\begin{array}{l}\text { Integrating Code } \\
\text { and Design }\end{array}$ & - & + \\
Mantenance & - & + \\
Portability & - & + \\
\hline Avallability & + & -
\end{tabular}

FIGURE 6: Comparison of the Two Case Techniques

\subsection{Design}

During the design phase, Today's CASE can be much simpler to use. The designer enters the design via the CASE provided graphical editor.

When employing CASE based on document generators the designer distinguishes between overview information and information that can be encapsulated directly in code. The designer must employ both the graphical editor to capture the design overview and an ASCII editor to capture component details. In order to avoid duplication between code and supplementary graphics, the overview diagrams are void of details.

Skeletal coding during the design phase is awkward, and the designer must resist the temptation to code. This skeletal coding is not truly coding but rather capturing the design knowledge directly into the code product.

There could be new CASE tools that address this problem such as a graphical editor, that supports direct modification of code.

\subsection{Implementation}

During implementation CASE based on document generators is easier to use. All details of the software components may be entered directly into code via an ASCII editor. Most implementers find ascii editors a simpler, quicker tool. The design is captured directly in code. The transition from design to implementation is seamless.

CASE based on code generators require detailed information to be entered via the CASE graphical tool. The implementer is encumbered to make numerous graphical decisions dictating the graphical position of the software component and the visibility of the component characteristics, e.g., methods and attributes.

CASE based on code generators maintains a distinction between design and code. For each design change the code must be regenerated. The constant overhead of code generation slows implementation. Maintaining consistency between the design and the code is a configuration management overhead. The developer tends to mistrust auto generated code.

Frustration frequently causes CASE based on code generators to be abandoned during implementation, permanently severing the link between design and code.

\subsection{Integrating Code and Design}

Reverse engineering techniques are provided with Today's CASE tools in order to document preexisting software. For Today's CASE tools, this documentation is separate from the rest of the system and is not easily integrated into the design document.

For CASE based on document generators all code forms the basis of the system description. The overview diagrams may have to reflect the integration of this component but the component description may be extracted via reverse engineering techniques. The description of preexisting software is readily integrated into the overall design.

If the link is severed between Today's CASE and code, it is difficult if not impossible to reconstruct. Code may at any time be integrated into the proposed CASE environment.

\subsection{Maintenance}

For CASE based on code generators, code changes must be performed via the CASE graphical editor. Failure to do 
so severs the design from the code. Resulting in documentation that does not reflect the actual code.

For CASE based on document generators, changes can be made directly to the code. The design document automatically reflects the changes via reverse engineering techniques. Changes to the overview document must be made only when a new component is identified or an old component is discarded or if the interaction between components is changed. Changes to the supporting graphics may be done, either before or after the code changes. The maintainer completes the maintenance action by regenerating the design document. Due to the strong dependence on reverse engineering techniques, the maintainer learns to rely on the accurate design document.

\subsection{Portability}

For CASE based on code generators the portability of the system is limited to the portability of the CASE tool. A standard CASE storage structure is a topic of discussion to solve this portability problem. To date there is no such standard.

For CASE based on document generators, much of the system can be ported directly since it is code. There are two minor limitations to porting this system:

1. The supporting graphics must be ported. And,

2. The encoded comments must be ported.

Porting the supporting graphics is a significantly smaller problem than porting an entire system.

The comment descriptions have been encoded indicating what they are e.g, data dictionary description, requirements. There is no standard for such an encoding, though such a standard is possible. Also translating the encoding from one form to another is an easily automated task.

\subsection{Avallability}

CASE tools based on code generators are readily available today. These same tools can be employed to implement the CASE based on document generator technique. Additionally tools such as consistency checking between code and supporting graphics are needed to complete the proposed CASE tool set.

\subsection{Future CASE Tool Capabllities}

The CASE tools required by CASE based on document generators is a simple set of tools. Most of which are already available from existing CASE based on code generators. Additional capabilities that would support the proposed CASE technique include:

$\checkmark$ Reverse engineering tools to provide additional graphical information.

$\checkmark$ Documentation generator to automate the interleaving of the supporting graphics and the reverse engineered descriptions into a cohesive design document

$v$ Consistency checker to report inconsistencies between the code and the supporting graphics

$\checkmark$ Code graphical editor to edit both the supporting graphics and the code.

$\checkmark$ A Complete CASE Tool - providing the capabilities of both CASE techniques.

Each of these proposed tools are briefly described in the following sections

\subsection{Reverse Engineering Tools}

A tool to generate a state transition diagram or a state table to graphically illustrate the encoded state machine would be a valuable addition to the reverse engineering tool set.

It is also possible to reverse engineer the overview diagrams. It is feasible to generate both Information Models and Data Flow Diagrams from code. Such a tool would allow the design to eventually be replaced by totally reverse engineere $d$ documentation.

\subsection{Documentation Generator}

A tool is needed to automate the interleaving of the supporting graphics with the reverse engineered descriptions. It should be simple to generate the design document. Regenerating the design document should be automated.

A minimal capability is available today via document tools such as FrameMaker. These document tools need the additional ability to import documents text and graphics via reference such that when the imported documents change, the design document automatically incorporates these changes. 
Within this paper, the documentation focus has been on a design document. There are other supporting documents such as Interface Control Documents and Operators Manuals that can be reverse engineered from the code. We have employed reverse eagineering techniques to generate both of these documents. More work is needed to refine these techniques.

\subsection{Consistency Checker}

This tool would compare the information in the supporting graphics with information in the code. Inconsistencies would be reported. Consistency checking is the strength of CASE tools. : Iven if overview diagrams are kept free of details they reflect information that is contained within the code. Also, these consistency checkers might be able to compare a state transition diagram or a state table with code and report discrepancies.

\subsection{Code Graphical Editor}

If the graphical editor of existing CASE tools, could be enhanced to edit code directly then the CASE based on document generators would evolve into a full CASE tool providing all the capabilities of both CASE techniques.

\subsection{A Complete CASE Tool}

The ultimate solution is to provide a CASE environment that integrates both of the comparative CASE techniques into a single CASE environment. This full CASE environment, illustrated on FIGURE 7: on page 9, would allow modification via either the graphical editor or directly to the code via an ascii editor. This ultimate CASE environment rould hide the internal storage of the CASE tools making it irrelevant as to whether the system was being stored internally or as code. Such a system would make code generators, if present, invisible to the developer.

There are two non-trivial problems that must be addressed before this propose CASE technique is realized:

$\checkmark$ Integrating the design reverse engineered from code with the preexisting design maintained within the CASE internal storage, and

$\checkmark$ Maintaining consistency between code and the design.

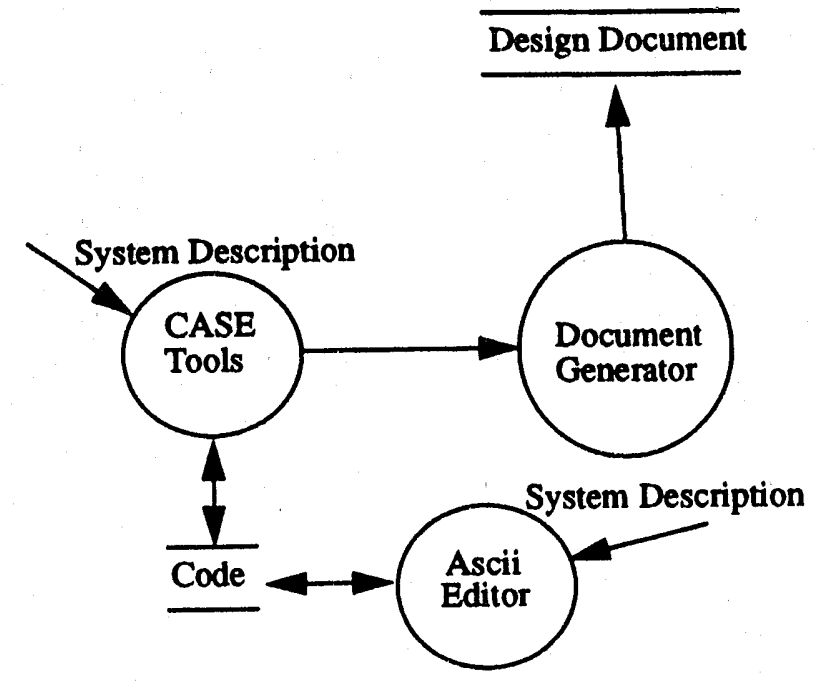

FIGURE 7: Full CASE capability supporting both Document and Code Generation

\subsection{Summary}

A CASE technique has been presented that offers:

$\checkmark$ Improved system portability,

$\checkmark$ Easier implementation, and maintenance, and

$\checkmark$ A seamless transition from design to code.

The proposed CASE technique is based on placing as much as possible of the system description directly in the code. The design document is generated from the code via reverse engineering techniques. Since most of the system is in code, the portability of the system is limited to the portability of the compiler. As long as there is a code Language Reference Manual but not a CASE standard, the "bible" defining the system must be the code. By basing the code as the "bible", the task of integrating new or modified code into the CASE environment is a simple task. This contrasts with the nearly irreparable severs between code and CASE that is typical of Today's CASE Tools.

Freedom from graphical editors and code generators simplify both the implementation and maintenance. The documentation maintenance task via the reverse engineering techniques results in documentation that accurately reflects the software system. 
The task of translating the design to code is eliminated as the boundary defined by code generators is erased. The system is the code. The design can be illustrated by applying reverse engineering techniques to the code. At any point the design may be changed. The changes are reflected directly in the code and/or supporting graphics. A Case consistency checking tool reports incc usistencies between the code and the supporting graphics.

Existing CASE tools may be used in a constrained fashion to implement this proposed CASE technique. Additional CASE capabilities, not readily available, could enhance this proposed CASE technique. Capabilities such as consistency checking between code and supporting graphics, and reverse engineering supporting graphics are realizable. A design document based on component descriptions reverse engineered out of code is proposed.

The proposed CASE based on document generators is a unique application of reverse engineering techniques to the design, development and documentation of new software systems.

\subsection{Acknowledgments}

I wish to thank Paul Attermeier, Louann Grady. John Rowe, and Larry Ellis, all of Sandia National Laboratories as well as Sue Spaven of Applied Physic Inc., all of whom assisted me in refining and clarifying this technique.

\subsection{References}

[1] Amelia Maxted, John C. Rowe, An Ada Graphical Tool to Support Software Development, Ada Letters, Using Ada: ACM SIGAda International Conference, Dec. 8-11, 1987.

[2] Sally Shlaer, Mellor, Object-Oriented Systems Analysis, Modeling the World in Data, 1988 PrenticeHall.

[3] Paul T. Ward, Object-Oriented Requirements Definition, Class presented by NTU Satellite Network on Feb. 11, 1991.

[4] FrameMaker, Using FrameMaker, Frame Technology Corporation, San Jose, California, October 1989., 


\section{Unlinulted Release}

1 8523-2 Ceatral Technical Files

57141 Technical Library

17151 Tectnical Publications

10 7613-2 Document Procesing for DOB/OSTI

50 232 Amelia Marted 

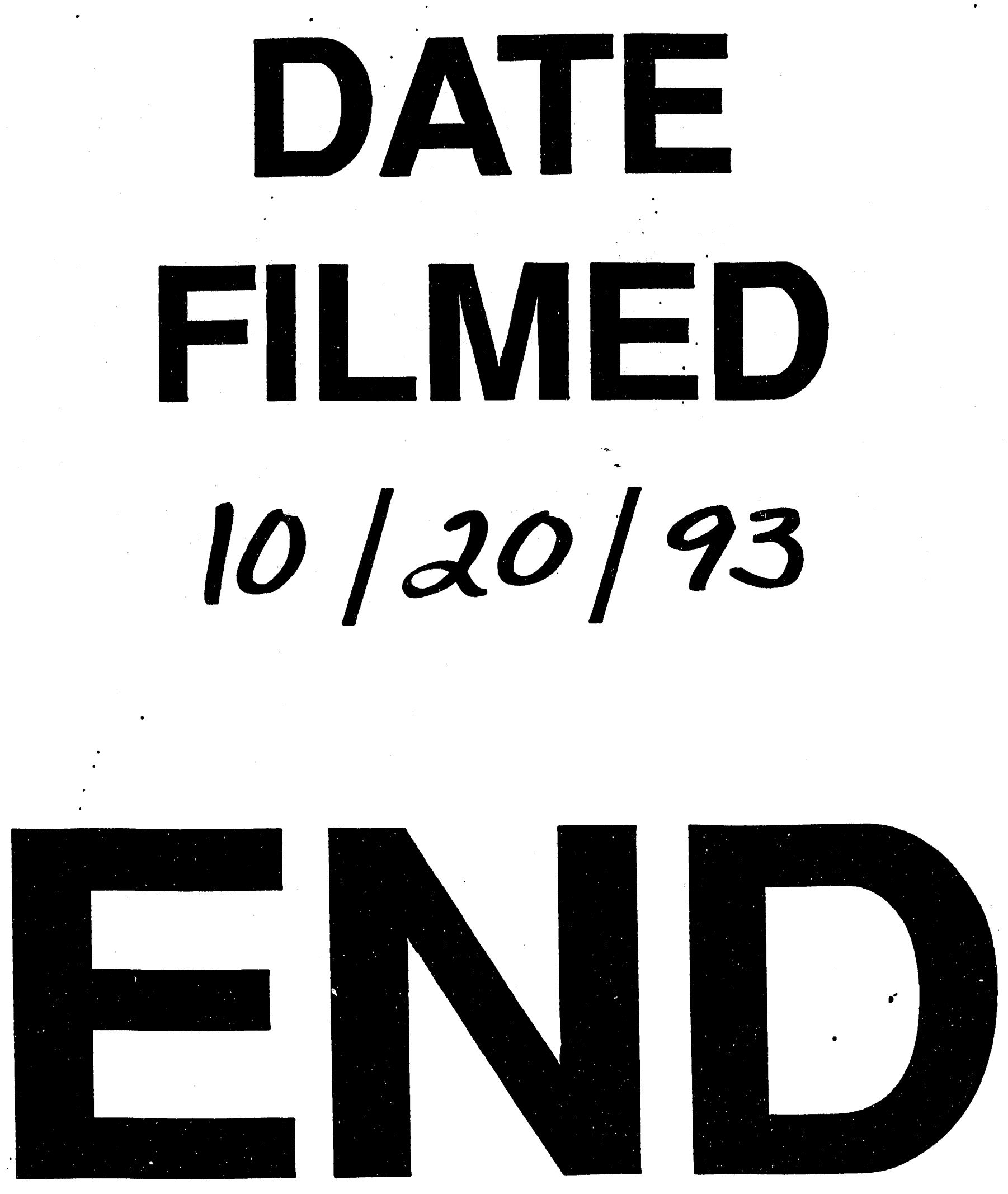
